\title{
Elaboración de planchas celu-plasticas a través del reciclaje de celulosa residual y tereftalato de polietileno (pet)
}

Fernando Albiño Velasco; Diego Mora Zurita; Diana Mosguidt Ramos Gladys Sobenis Hinojosa; Carlos Velasco Sánchez; Lenin Arellano Barragán

Universidad Estatal de Bolívar fernando-velasco19@hotmail.com

\section{Resumen}

En la búsqueda de presentar un producto sustituto con mejores cualidades que la madera natural, se decidió producir madera plástica por medio de la combinación de celulosa y polietileno de tereftalato (PET). Dentro de la Provincia Bolívar, Cantón San Miguel, la principal fuente de ingreso es la agricultura, la cual genera grandes cantidades de desechos vegetales los que no se han expuesto a un adecuado proceso de eliminación, considerando también que dentro del medio existe grandes cantidades de desechos generados por productos de consumo masivo aumentando el nivel de contaminación. Generar un nuevo producto y bajar los niveles de contaminación en el medio son el propósito fundamental, es por esto que se ha combinado estos dos tipos de desechos: celulosa residual y polietileno de tereftalato a través del método de exposición al calor con la cual se origina una plancha celu-plástica mucho más resistente a inclemencias ambientales, pudiendo industrializarse.

Palabras clave: Planchas celu-plásticas, celulosa, polietileno de tereftalato (PET).

\section{Abstract}

In San Miguel, located in the Bolivar Province, Ecuador, the principal source of incomes comes from agricultural activities causing a great deal of vegetables wastes which are not adequately eliminated, considering as well, other great quantity of plastic wastes derived from the mass produced consumer products which elevate the levels of contaminations. With the purpose of presenting a new commodity with better qualities which may substitute the timber, and can diminish the levels of pollution, the authors of this paper manufactured a kind of plastic timber using the mixture of cellulose and polyethylene terephthalate (PET) which are exposed to intensive heat generating, as a result, a cellulose plastic plate more flexible and weather resistant able to be used in the construction industry.

Keywords: Cellulose plastic plate, cellulose, polyethylene terephthalate (PET).

Cómo citar este artículo: Albiño F, Mora D., Mosguidt D., Sobenis G., Velasco C. y Arellano L. (2015) Elaboración de planchas celu-plasticas a través del reciclaje de celulosa residual y tereftalato de polietileno (pet). CUMBRES, Revista Científica. 1(2) 55 - 58 


\section{Introducción}

La principal causa de contaminación ambiental en el Cantón San Miguel de la Provincia Bolívar es producida por los propios habitantes, no tienen conciencia de que la combustión de desechos agrícolas, así como el arrojar residuos plásticos afecta el ambiente natural que los rodea- dañando su salud y vida. Procesar residuos de celulosa y polietileno como solución alternativa a la contaminación existente en el medio es por lo que se desarrolla este proyecto.

San Miguel de Bolívar es uno de los mayores productores de trigo, cebada, maíz, donde se encuentra un sin número de productos vegetales, que originan una significativa variedad de desechos, el aprovechar estos como materia prima para nuestros propósitos, sería ideal, por lo que se convierten en materia prima esencial para nuestro objetivo, es decir, utilizamos estos desechos constituidos de celulosa y hemos considerado además, el uso de los desechos plásticos que se emplean para la conservación de los alimentos los cuales son incinerados como práctica tradicional a campo abierto.

La mayor parte de los habitantes no tienen conocimiento que estos productos emiten gases tales como el Metano (CH4), Monóxido de carbono (CO), bióxido de nitrógeno (NO2), Hidrocarburos ( $\mathrm{CnH} 2 \mathrm{n}+2)$ y partículas menores a 10 micras (PM10)2, alguna de las cuales forman parte de los gases que producen el efecto invernadero (calentamiento global).

La emisión de humo producido por estos procesos genera problemas respiratorios e irritaciones en los ojos a toda la población que esté expuesta, además de emitir dioxinas y furanos compuestos que son muy dañinos para la salud humana, animal y vegetal, considerados como uno de los causantes del cáncer, es por ello que nos hemos visto en la necesidad de darle a estos desechos un uso adecuado, y bajo un tratamiento de reciclaje convertirlos o reutilizarlos en un producto nuevo, o sea, mediante métodos innovadores transformarlo en un material alternativo con cualidades de resistencia e impermeabilidad, como es la madera plástica o- como la denominaremos en esta investigación por las características de su composición- planchas celu-plásticas, para la construcción de las cuales utilizaremos por sus magníficas propiedades físicas, los compuestos como el polietileno de tereftalato y celulosa residual de los desechos del maíz, la cebada, trigo y productos vegetales que contienen celulosa- un tipo de polímero al igual que el PET por lo cual estos materiales se pueden combinar sin complicación.
Se persigue crear planchas celu-plásticas, las cuales reemplazarán a la madera natural evitando la tala de bosques del sector y disminuyendo la contaminación por el acumulamiento y eliminación inadecuada.

\section{Materiales y métodos}

Se consideró emplear residuos agrícolas tales como la calcha de maíz, la paja de trigo y cebada ya que se encuentran en el medio abundantemente y los costos para la adquisición de estos materiales no sería nada elevado, se pensó en la utilización de estos por tener un alto contenido de celulosa y por encontrarse en el medio en gran proporción secos.

También se consideró emplear el PET de botellas plásticas de agua, yogurt y gaseosas las cuales son clasificadas por color y textura identificando el grosor de las mismas.

Para (García y col., 2013) "un material compuesto es aquel que se forma al mezclar dos o más materiales distintos, sin que haya reacción química. El material compuesto presenta dos elementos principales: un refuerzo y una matriz." (p.29) En este caso de los materiales elegidos la matriz actúa como elemento que articula y da consistencia (el polietileno de tereftalato (PET) y uniformidad, mientras que el refuerzo otorga la resistencia- la celulosa residual.

Para poder procesar los dos materiales anteriormente expuesto, se identificó los componentes que forman cada uno de estos, es decir el nivel de celulosa que contienen los desechos agrícolas y el nivel de PET que contienen cada uno de los envases y así poder determinar las cantidades necesarias para su combinación.

Se realizaron pruebas de picado, secado y exposición a calor para identificar el proceso idóneo para la combinación de estos dos componentes, dentro de los cuales estuvieron la clasificación tanto de celulosa como de polietileno de mayor y menor densidad al igual que el picado de los mismos, luego se procedió a exponer el polietileno a diversas temperaturas desde $120^{\circ} \mathrm{C}$ hasta $300{ }^{\circ} \mathrm{C}$, pudiendo determinar que la temperatura para lograr el punto de fusión, es de 260 a $265^{\circ} \mathrm{C}$ en exposición continua combinado con movimiento, punto en el cual se agrega la celulosa.

Los desechos agrícolas fueron secados completamente dentro de una máquina de secado de vegetales, ya que estos a pesar de estar secos conservan algo de humedad en su interior, una vez secos se procedió a triturarlos, en las pruebas que realizamos esta trituración se la hizo con molinos normales de grano pero no dio resultado, ya que

\section{Cumbres}


debía haber mucha más fuerza, por lo cual se decidió diseñar una maquina picadora la cual además de picar estos desechos los hace prácticamente polvo y así con esto se garantiza que se compacte de una mejor forma el PET con la celulosa.

Una vez triturada la paja se procedió a la clasificación del PET, es decir por color y dejando de lado la parte superior y las tapas, ya que en esta parte de la tapa tiene mayor dureza por lo que impide que el punto de fusión se obtenga al mismo tiempo que lo demás, se consideró separar por colores el PET, para así poder tener una buena imagen en el producto, una vez clasificados se ingresan a la misma picadora para agilizar el proceso de volver liquido al PET.

El PET picado se expuso al calor continuo de 260 a $265^{\circ} \mathrm{C}$, para que el PET se vuelva líquido y mantenerlo así, se lo mantuvo en movimiento donde se va agregando la celulosa picada, se traspasa al molde rectangular para así poder dar una forma de planchas por lo menos de 20 centímetros por 15 centímetros, este molde está expuesto al calor combinado con presión para que tome esta forma.

El proceso de enfriado se realizó introduciendo este molde que contiene el producto prensado en agua fría, produciéndose así un choque térmico con lo cual se garantiza que nuestro producto asuma la forma deseada mucho más rápido pero se dio origen a un producto algo quebradizo. Por lo cual se consideró que al estar prensado se lo deje enfriar, a temperatura ambiente.

\section{Resultados}

Mediante la utilización de celulosa residual y PET reciclado, se obtuvo un nuevo material muy resistente como son planchas celu-plásticas, con la combinación de componentes que son físicamente compatibles, se dio un producto nuevo con materiales reciclados reutilizados, innovadores y prácticamente amigables con el medio ambiente.

Se determina que al conseguir un mesclado idóneo entre el PET y la celulosa se pudo obtener un producto muy resistente a la humedad, presión; siendo este un producto altamente competitivo por sus cualidades y versatilidad en sus usos.

Con la industrialización se podrá dar un mayor realce al entorno social, ya que esto ayudará a su desarrollo mediante la creación de nuevas fuentes de trabajo, por lo que representa una gran contribución con la sociedad.

Se logra también la disminución de la contaminación al re- utilizar este tipo de materiales contribuiremos a que se utilicen nuevos productos alternativos y de esta forma disminuir la tala indiscriminada de nuestros recursos naturales, y la no incineración de productos plásticos que son los mayores causantes de contaminación de nuestro aire.

Prácticamente este producto, asegura su gran aceptación dentro del mercado logrando así obtener recursos económicos propios, para el continuo desarrollo de nuestra industria, con estos recursos lograremos mejorar e innovar las aplicaciones que se podrán dar, al igual que se implementarán nuevas técnicas para la transformación de este material.

Generar nuevas fuentes de trabajo, disminuir la contaminación, bajar el nivel de destrucción de nuestros bosques, son los principales fines de este proyecto de mucha importancia.

\section{Discusión}

Se comprobó que existen otros estudios e investigaciones sobre el tema, pero no encontramos en las consultadas ninguna que utilizaran exactamente las mismas materias primas, ya que ellas dependen en gran medida de optimizar los recursos del contexto y en nuestro caso además, evitar la tala indiscriminada de nuestros recursos forestales reutilizando desechos agrícolas abundantes en nuestro contexto natural. (García y col., 2013), (París y col., 2009), (Solís y col., 2002).

Por consiguiente, la generación de este nuevo producto alternativo se enfoca a sustituir las necesidades en la utilización de productos a base de la madera natural, que se ha utilizado por mucho tiempo afectando nuestro medio natural reemplazándola por las planchas celu-plásticas, ya que ofrece mucha más resistencia, durabilidad e incluso impermeabilidad, por lo que tendrá una mejor acogida en cualesquier ámbito que utilicen las planchas celu-plásticas.

\section{Conclusiones}

1. Con la creación de nuevas fuentes de trabajo, se podrá combatir uno de los principales factores que influyen mucho en la sociedad, como es el desempleo y de esta forma contribuir; que en el medio se cumpla con los nuevos lineamientos del "Buen Vivir", el cual se enfoca en que todos y cada uno de los integrantes de nuestra sociedad cuenten con los recursos y medios necesarios para poder satisfacer sus necesidades.

2. Uno de los principales factores que influyen en la 
contaminación, es la incineración tanto de residuos agrícolas como de materiales plásticos, por lo que con la reutilización de estos materiales estaremos contribuyendo con la disminución de contaminación medio ambiental, por lo que es muy viable realizar la combinación de estos materiales para darles un nuevo uso, mediante la transformación y la obtención de un producto alternativo.

3. Con los recursos económicos obtenidos no solo se podrá poner en marcha una nueva adecuación de esta micro industria, sino que igualmente se apoyará al desarrollo social y económico del poblado de San Miguel, contribuyendo potencialmente con las comunidades aledañas, manteniendo la colaboración mutua entre las partes involucradas en este acontecimiento industrial y comercial.

4. La difusión de este nuevo método de reciclaje para transformarlo en tablas celu-plásticas es el propósito y resultado de nuestro trabajo, y con ello poder impulsar una campaña de reciclaje que viabilice la disminución de la contaminación, creando esta nueva alterna- tiva posibilitamos la obtención de recursos reciclados del propio medio que son más perdurables, por tanto más económicos para las familias que habitan en el entorno, mejorando así su calidad de vida.

\section{Referencias bibliográficas}

- García, A., et al. (2013). Madera Plástica con paja de trigo y matriz polimérica. Rev. Tecnología en Marcha, Vol. 26, No. 3. Disponible en: www.dialnet.unirioja.es/ servlet/articulo?codigo $=4835722$

- Solís, M., y Lisperguer, J. (2002). Compuestos mixtos a base de plásticos y madera. Rev. Theoria, Vol. 11. Disponible en: www.ubiobio.cl/theoria/v/v11/5.pdf

- París, L. y González, S. (2002). Caracterización de los materiales plásticos reciclados provenientes de la industria bananera empleados para la elaboración de madera plástica. Suplemento de la Revista Latinoamericana de Metalurgia y Materiales. Disponible en internet: www. rlmm.org/archives. php?f=/archivos/S01/N4/RLMMArt... p1453

\section{$58 \quad$ Cumbres}

